This is an open access article distributed under the terms of the Creative Commons BY-NC-ND Licence

\title{
Cloning, characterization, and expression of 1-deoxy-D-xylulose-5- phosphate reductoisomerase gene from Dioscorea zingiberensis
}

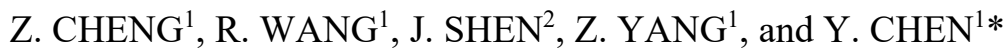 \\ Department of Biotechnology and State Key Laboratory of Biocatalysis and Enzyme Engineering, \\ School of Life Sciences, Wuhan 430062, Hubei Province, P.R. China ${ }^{1}$ \\ School of Basic Medical Sciences, Hubei University of Medicine, Shiyan 442000, Hubei Province, P.R. China ${ }^{2}$
}

\begin{abstract}
Diosgenin, mainly extracted from some Dioscorea species, is the most important starting material for the production of steroidal drugs. It is believed that diosgenin in Dioscorea is synthesized from isopentenyl diphosphate (IPP) and dimethylallyl diphosphate (DMAPP, the isomer of IPP) produced by the cytosolic mevalonate pathway. So far, the possibility of the plastidic 2-C-methyl-D-erythritol 4-phosphate (MEP) pathway for biosynthesis of diosgenin remains unclear. The key enzyme in the MEP pathway is 1-deoxy-D-xylulose 5-phosphate-reductoisomerase (DXR, EC: 1.1.1.267). In this study, a DXR gene, named DzDXR (GenBank accession number KY131955), was isolated from Dioscorea zingiberensis. The DzDXR has an open reading frame of 1413 bp encoding a protein of 470 amino acid residues. The function of $D z D X R$ was verified by a colour enhancement assay in the Escherichia coli cells harbouring the plasmid pAC-BETA. Bioinformatic analyses revealed that DzDXR had a putative plastid transit peptide at the N-terminal region and was highly homologous to other plant DXRs, especially to those in monocotyledons. During the growth period of $D$. zingiberensis, the expression of $D z D X R$ was found significantly high in leaves and very low in tubers, and the highest expression was observed in mature leaves in summer. In contrast to the $D z D X R$ transcription, diosgenin was present predominantly in tubers and in minute quantities in leaves. Because diosgenin is very likely formed mainly in the cytosol of mature leaf cells of Dioscorea and the plastidic IPP and DMAPP produced by the MEP pathway can be transported into the cytosol, the consistently high expression of $D z D X R$ detected in mature leaf of $D$. zingiberensis implies that the MEP pathway might play a significant role in diosgenin biosynthesis.
\end{abstract}

Additional key words: $\beta$-carotene, diosgenin biosynthesis, Escherichia coli, MEP pathway, plasmid pAC-BETA.

\section{Introduction}

Diosgenin has multiple biological activities and has been used for production of numerous steroidal drugs for more than half a century (Patel et al. 2012, Chen et al. 2015). Although diosgenin occurs in many plants, it is mainly obtained from some Dioscorea species. To date diosgenin biosynthetic pathway is not fully known. Isotope feeding studies have proved that cholesterol is the precursor of diosgenin in Dioscorea (Bennett et al. 1965, 1970, Joly et al. 1969a,b, Stohs et al. 1969) and Dewick (2009) proposed a pathway from cholesterol to diosgenin. Cholesterol is one of phytosterols that exist ubiquitously in plants. Phytosterols are synthesized in the cytosol from isopentenyl diphosphate (IPP) and its isomer dimethylallyl diphosphate (DMAPP) (Piironen et al. 2000, Suzuki and Muranaka 2007).

Submitted 22 June 2018, last revision 11 October 2018, accepted 15 October 2018.

Abbreviations: DMAPP - dimethylallyl diphosphate; DXP - 1-deoxy-d-xylulose 5-phosphate; DXR - 1-deoxy-d-xylulose 5-phosphate reductoisomerase; IPP - isopentenyl diphosphate; MEP - 2-C-methyl-D-erythritol 4-phosphate; ORF - open reading frame; qPCR quantitative polymerase chain reaction; RACE - rapid amplification of cDNA ends.

Acknowledgements: This work was financially supported by a project (No. 2014CFA081) from the Department of Science and Technology of Hubei Province, China. We gratefully acknowledge Dr. Francis X. Cunningham (Department of Cell Biology and Molecular Genetics, University of Maryland, USA) for kindly providing the plasmids pAC-BETA and pTrcAtIPI and Dr. Yushan Long (Pan Agriculture, Sydney, Australia) for revising the language of the manuscript.

The first two authors contributed equally to this work.

* Corresponding author; e-mail: chenyongqin@hubu.edu.cn 
Plants have two independent pathways for production of IPP and DMAPP, the mevalonate pathway in cytoplasm and the 2-C-methyl-D-erythritol 4-phosphate (MEP) pathway in plastids. Traditionally, it is believed that these two pathways are strictly compartmentalized: isoprenoids such as phytosterols, triterpenes, and sesquiterpenes are from the cytosolic IPP and DMAPP, while other isoprenoids like isoprene, monoterpenes, diterpenes, tetraterpenes, carotenoids, phytols, tocopherols, and plastoquinone are from the plastidic IPP and DMAPP (Lichtenthaler 1999, Bick and Lange 2003, Hemmerlin et al. 2012, Vranová et al. 2013). Based on these findings, it is concluded that diosgenin is derived from the cytosolic IPP and DMAPP. However, the crossflow between the two pathways has been reported in some plants like Antirrhinum majus (Dudareva et al. 2005), Arabidopsis thaliana (Laule et al. 2003), Catharanthus roseus (Schuhr et al. 2003), Coluria geoides (Skorupinska-Tudek et al.

\section{Materials and methods}

Dioscorea zingiberensis C.H. Wright plants were collected in Shiyan, Hubei Province, China, and grown in the field at Hubei University, Wuhan, China. Total RNA was extracted from the young leaves using TRIzol reagents (Invitrogen, Carlsbad, CA, USA) and then reverse transcribed into cDNA using $M-M L V$ RTase cDNA synthesis kit (TaKaRa, Dalian, China) according to the user's manual.

Cloning of full length cDNA of $D$. zingiberensis DXR gene $(\boldsymbol{D} \boldsymbol{z} \boldsymbol{D} X \boldsymbol{R})$ : Degenerate oligonucleotide primers DXR-F and DXR-R (Table 1 Suppl.) were designed according to the conserved regions of known plant DXRs to amplify a partial cDNA fragment of $D$. zingiberensis $D X R$ gene. Degenerate PCR was conducted in a volume of $20 \mathrm{~mm}^{3}$ containing $14 \mathrm{~mm}^{3}$ of $\mathrm{ddH}_{2} \mathrm{O}, 2 \mathrm{~mm}^{3} 10 \times$ TransTaq HiFi II (TranGen Biotech Co., Beijing, China) buffer, $2 \mathrm{~mm}^{3}$ of $10 \times G C$ enhancer, $0.4 \mathrm{~mm}^{3}$ of $10 \mathrm{mM}$ dNTP mix, $0.4 \mathrm{~mm}^{3}$ cDNA, $0.4 \mathrm{~mm}^{3}$ of $10 \mu \mathrm{M}$ DXR-F, $0.4 \mathrm{~mm}^{3}$ of $10 \mu \mathrm{M}$ DXR-R, and $0.4 \mathrm{~mm}^{3}$ of TransTaq HiFi DNA polymerase under the following conditions: $94{ }^{\circ} \mathrm{C}$ for $3 \mathrm{~min}$, then 35 cycles of $94{ }^{\circ} \mathrm{C}$ for $30 \mathrm{~s}, 50{ }^{\circ} \mathrm{C}$ for $30 \mathrm{~s}$ and $72{ }^{\circ} \mathrm{C}$ for $1 \mathrm{~min}$, and a final extension at $72{ }^{\circ} \mathrm{C}$ for 10 min. Based on the partial cDNA sequence, primers DXRGSP1 and DXR-GSP2 (Table 1 Suppl.) were synthesized to clone the $5^{\prime}$ and 3 ' ends of the cDNA fragment by rapid amplification of cDNA ends (RACE), respectively. The RACE PCR was performed using $S M A R T^{T M}$ RACE cDNA amplification kit (Clontech, Mountain View, CA, USA) following the manufacturer's instructions. The full-length cDNA of $D$. zingiberensis $D X R$ gene, designated $D z D X R$, was obtained by assembling the partial cDNA fragment and the $5^{\prime}$ and $3^{\prime}$ end sequences via ContigExpress software. The intact open reading frame (ORF) of $D z D X R$,
2008), Salvia miltiorrhiza (Yang et al. 2012a), tobacco (Hemmerlin et al. 2003), and tomato (Gutensohn et al. 2013). Moreover, it has been found that the transport of isopenoid precursors from the plastids to the cytosol is more effective (Bick and Lange 2003, Schuhr et al. 2003). So far, whether the MEP pathway is involved in diosgenin biosynthesis in Dioscorea is unclear. Clarifying this issue is not only scientifically interesting but also important for breeding elite cultivars of Dioscorea. Cloning the MEP pathway genes of Dioscorea will help us to investigate this issue using molecular techniques. 1-deoxy-D-xylulose5-phosphate reductoisomerase (DXR; EC 1.1.1.267) catalyzes the formation of MEP from 1-deoxy-D-xylulose 5-phosphate (DXP), a committed step in the MEP pathway (Carretero-Paulet et al. 2002, Hasunuma et al 2008). In this paper, we report cloning, characterization, and expression analysis of a $D X R$ gene from $D$. zingiberensis, which is cultivated in China for diosgenin production.

named DzDXR-ORF, was amplified with primers DZR(ORF)-F and DXR(ORF)-R (Table 1 Suppl.) using a program of pre-denaturation at $94{ }^{\circ} \mathrm{C}$ for $3 \mathrm{~min}$, followed by 35 cycles of at $94{ }^{\circ} \mathrm{C}$ for $30 \mathrm{~s}$, at $59{ }^{\circ} \mathrm{C}$ for $30 \mathrm{~s}$, and at $72{ }^{\circ} \mathrm{C}$ for $90 \mathrm{~s}$, and an extension of at $72{ }^{\circ} \mathrm{C}$ for $8 \mathrm{~min}$.

The above amplification products, e.g., the partial cDNA fragment, 5' and 3' end cDNA, and DzDXR-ORF, were inserted into pMD18-T vector (Promega, Madison, WI, USA) and transformed into competent Escherichia coil (strain TOP10). In each transformation, at least five positive recombinant clones were sequenced.

Bioinformative and functional analysis of $D_{z} D X R$ : The ORF of $D z D X R$ was identified and the amino acid sequence was deduced by ORF Finder on NCBI (http://www.ncbi.nlm.nih.gov/gorf/gorf.html). Multiple sequence alignment of DXRs was conducted using the $D N A M A N$ program. The presence of signal peptides was predicted by using the TargetP 1.1 server (http://www.cbs. dtu.dk/services/TargetP-1.1) and the ChloroP 1.1 server (http://www. cbs.dtu.dk/ services/ChloroP/).

Plasmids pAC-BETA and pTrcAtIPI were used to test the function of $D z D X R$. The pAC-BETA contains a chloramphenicol resistance gene and four functional genes necessary for $\beta$-carotene biosynthesis, $\operatorname{crt} E, \operatorname{crt} B, \operatorname{crt} L$, and $c r t Y$. The pTrcAtIPI contains an ampicillin resistance gene and an AtIPI gene.

After the vector containing $D z D X R$-ORF was digested with Pst I and Not I DzDXR-ORF was recovered and subcloned into the plasmid pTrcAtIPI that was digested by the same enzymes to remove the insert AtIPI, creating a recombinant plasmid pTrc-DzDXR. The pTrcAtIPI was also digested by Pst I to remove AtIPI and then self-ligated by T4 DNA ligase to yield a control vector pTrc. Both pTrc-DzDXR and pAC-BETA were co-transformed into 
competent E. coli (strain TOP10F'). The E. coli TOP10F' cells were also transformed with plasmid pTrc alone or together with plasmid pAC-BETA and used as controls. The transformants containing both pTrc-DzDXR and pAC-BETA or both pTrc and pAC-BETA were cultured on solid Lurie and Broth (LB) medium supplemented with $50 \mathrm{mg} \mathrm{dm}^{-3}$ chloramphenicol and $100 \mathrm{mg} \mathrm{dm}^{-3}$ ampicillin, and the transformants containing pTrc were cultured on solid LB medium with $100 \mathrm{mg} \mathrm{dm}^{-3}$ ampicillin at $37^{\circ} \mathrm{C}$. Single colonies of the above transformants and wild type E. coli TOP $10 \mathrm{~F}^{\prime}$ cells were cultured in $2 \mathrm{~cm}^{3}$ liquid LB medium at $37^{\circ} \mathrm{C}$ for $1 \mathrm{~d}$. These cultures were streaked on an antibiotic free LB agar plate and incubated at $37^{\circ} \mathrm{C}$ for $1 \mathrm{~d}$ and at $28{ }^{\circ} \mathrm{C}$ for $2 \mathrm{~d}$. The function of $D z D X R$ was evaluated based on the colour of the transformants.

Expression analysis of $D z D X R$ in $D$. zingiberensis: $D z D X R$ transcriptions in $D$. zingiberensis were analyzed using real-time quantitative (q)PCR. The young leaves, mature leaves, newly formed tubers (young tubers), and old tubers were collected from three field-grown plants in the middle of April, July, and September 2017. Total RNA was extracted and cDNAs were synthesized as mentioned above. The housekeeping gene glyceraldehyde 3-phosphate dehydrogenase $(G A P D H)$ was used as an internal control. The primer pair DzDXR(RT)-F and DzDXR(RT)-R and the primer pair GAPDH-F and GAPDH-R (Table 1 Suppl.) were used to amplify the transcripts of $D z D X R$ and $G A P D H$, respectively. The PCR reactions were performed using SYBR Premix ExTaq ${ }^{\mathrm{TM}}$ (Tli RNaseH Plus) (Takara) on the Bio-Rad CFX96 ${ }^{\mathrm{TM}}$ real-time PCR system. The conditions were: $95{ }^{\circ} \mathrm{C}$ for $3 \mathrm{~min}, 40$ cycles of $95{ }^{\circ} \mathrm{C}$ for $15 \mathrm{~s}$ and $60{ }^{\circ} \mathrm{C}$ for $30 \mathrm{~s}$, and a final dissociation stage at $95^{\circ} \mathrm{C}$ for $15 \mathrm{~s}, 60^{\circ} \mathrm{C}$ for $1 \mathrm{~min}$, and $95^{\circ} \mathrm{C}$ for $15 \mathrm{~s}$. Triplets of all the samples were run, and the specificity of each primer pair was validated by a dissociation curve. The Pfaffl (2001) method was used to calculate the relative expression of $D z D X R$. Because young tubers in April and young leaves in September were not available, data on $D z D X R$ expression in these tissues were absent.

Diosgenin determination: Leaves and tubers were harvested from three plants in late October. Old and young tubers were separated and sliced. All the samples were dried at $60{ }^{\circ} \mathrm{C}$ and then grounded to powder. Diosgenin extraction was done as described by Mou et al. (2015) with some modifications. Briefly, about $100 \mathrm{mg}$ of the powder was added into a $50 \mathrm{~cm}^{3}$ flask containing $20 \mathrm{~cm}^{3}$ of $1 \mathrm{M}$ sulfuric acid and kept at $125^{\circ} \mathrm{C}$ for $3 \mathrm{~h}$ for hydrolysis. The hydrolysate was filtered and washed with distilled water until it became neutral. The hydrolysate together with the filter paper was extracted three times with petroleum ether and dried under vacuum on a rotary evaporator. The petroleum ether extract was dissolved in $2 \mathrm{~cm}^{3}$ methanol and filtered before subjected to analysis. Reverse-phase high performance liquid chromatography (HPLC) was employed for quantitative determination of diosgenin. The chromatographic analysis was performed on an Agilent 1100 HPLC system (Agilent Technologies, Palo Alto, CA, USA) using water + methanol $(10: 90, \mathrm{v} / \mathrm{v})$ as the mobile phase with a flow rate of $1 \mathrm{~cm}^{3} \mathrm{~min}^{-1}$ and detected at 210 nm (Huang et al. 2008). A calibration curve was generated with diosgenin standard (Sigma-Aldrich, St. Louis, USA) and diosgenin content was calculated by the method of external standards.

Data analysis: Data on $D z D X R$ transcription and diosgenin content were statistically analyzed by $A N O V A$ and Tukey's test at $1 \%$ significance using IBM SPSS program.

\section{Results}

A specific, 773-bp cDNA fragment was amplified by degenerate PCR. Then, a 5' end of $807 \mathrm{bp}$ and a 3' end of 956 bp were obtained by RACE PCR. Finally, a full length cDNA of 1643 bp was generated by assembling the partial cDNA fragment and its 5' and $3^{\prime}$ end sequences, which was designated DzDXR (GenBank accession number KY131955). The $D z D X R$ has an ORF of $1413 \mathrm{bp}$, a $5^{\prime}$ untranslated region (UTR) of $42 \mathrm{bp}$ and a $3^{\prime}$ UTR of $188 \mathrm{bp}$. The intact ORF of $D z D X R$ was obtained by PCR. Sequencing results of all the PCR products indicated that only one $D X R$ gene was identified.

$D z D Z R$ encodes a polypeptide of 470 amino acid residues. BLAST analysis of DzDXR at the $N C B I$ server revealed that DzDXR shared a high sequence similarity with other plant DXRs, especially with those of monocotyledons, for examples, $87 \%$ identity to Lilium longiflorum DXR (AHJ57307) and $86 \%$ identity to
Narcissus tazetta (ADD82536), Oryza sativa (AAL37560), Sorghum bicolor (XP_021311303), and Zea mays (XP_008655547) DXRs. Multiple sequence alignment of DzDXR, E. coli DXR, and other plant DXRs is shown in Fig. 1 Suppl.. Like other plant DXRs, DzDXR has an extension at the N-terminal side that is not present in the E. coli DXR. The extension is poorly conserved but enriched in Ser residues, which is thought as the typical features of plastid transit peptides (Heijne et al. 1989), and contains a Pro rich region defined as P(P/Q)PAWPG(R/T)A motif (Carretero-Paulet et al. 2002). Data analysis with both the TargetP 1.1 program and the ChloroP program predicted that DzDXR has a plastid localization signal in the first 46 amino acids. Based on the predicted value, the putative cleavage site of DzDXR (Fig. 1 Suppl.) was at the Cys-Ser-Met motif, which is consistent with the prediction of Carretero-Paulet 
et al. (2002) that the processing site of plant DXRs is at the $\mathrm{N}$ terminus of a conserved Cys-Ser-X motif ( $\mathrm{X}$ is any of the hydrophobic residues Ala, Val, or Met). The two conserved NADPH binding motifs [GSTGS(I/V)GT and LAAGSN(V/I)] and the two conserved substrate (DXP) binding motifs (LPADSEHSAI and NKGLEVIEAHY) found in other DXRs were also present in DzDXR (Fig. 1 Suppl.).

IPP and DMAPP are also the precursors for carotenoids. E. coli has the MEP pathway producing IPP and DMAPP, but cannot synthesize carotenoids due to lack of other genes necessary for carotenoid biosynthesis. After the plasmid pAC-BETA is introduced into E. coli, the transformed cells can produce $\beta$-carotene and have a yellow colour (Cunningham et al. 1996, Cao et al. 2010). If $D z D X R$ encodes a functional DXR, the E. coli cells harbouring both pAC-BETA and pTrc-DzDXR can produce more IPP and DMAPP and then more $\beta$-carotene, and therefore should be yellower than those bearing pAC-BETA or both pAC-BETA and pTrc. As expected, the cells co-transformed with pAC-BETA and pTrcDzDXR were obviously yellower than the control cells containing both pAC-BETA and pTrc (Fig. 1A,C), demonstrating that $D z D X R$ is a functional gene. Like the wild type cells, the cells transformed with the vector pTrc were white as they were unable to synthesize carotenoids (Fig. 1B,D).

The qPCR analysis of $D z D X R$ transcripts showed that during the growth period of $D$. zingiberensis, $D z D X R$ expressions had a similar expression patterns in different organs (Fig. 2A). Regardless of seasons, $D z D X R$ was expressed highly in mature leaves, moderately in young leaf, and lowly in tubers. The expression of $D z D X R$ in mature leaf was much higher in July than in April and September, indicating that $D z D X R$ expression in leaves was related to the seasons. Unlike $D z D X R$ transcription, most of diosgenin was present in tubers, especially in old tuber (Fig. 2B). The average content of diosgenin in the old tubers, young tubers, and mature leaves were 67.4, 51.3, and $8.6 \mathrm{mg} \mathrm{g}^{-1}$ (d.m.), respectively.

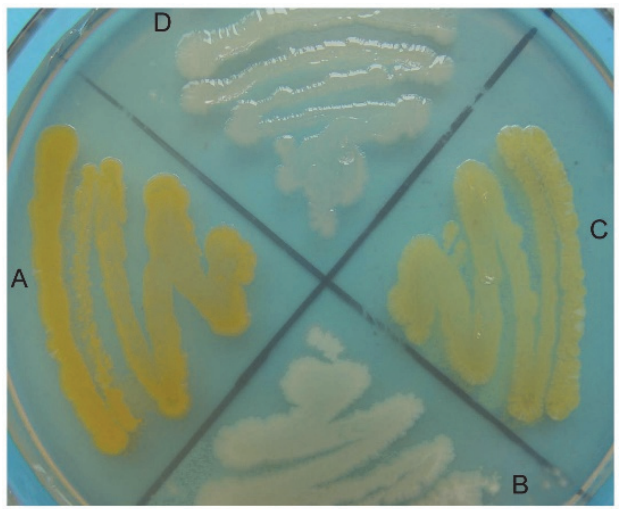

Fig. 1. Functional demonstration of $D z D X R$ in E. coli cells. $A$ - transformants containing pAC-BETA and pTrc-DzDXR; $B$ - wild type cells ; $C$ - transformants containing pAC-BETA and $\mathrm{p}$ Trc; $D$ - transformants containing only $\mathrm{p}$ Trc.
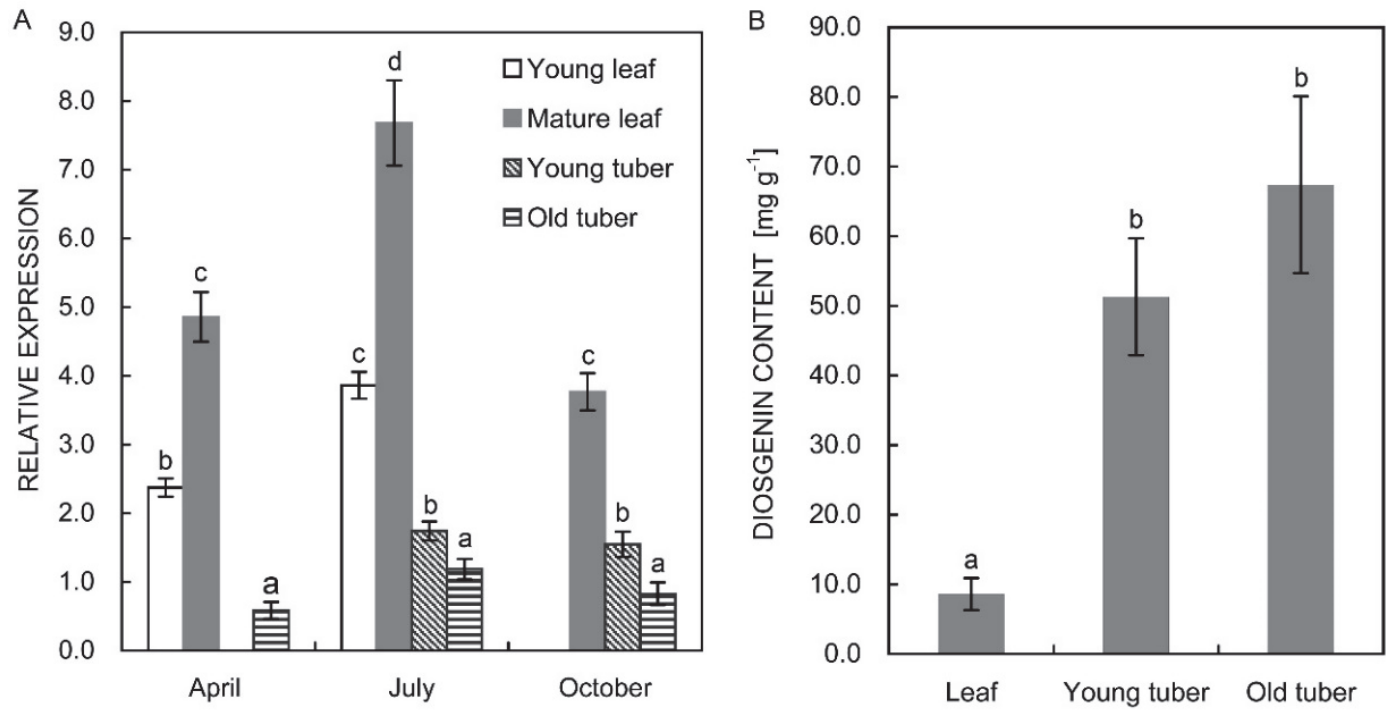

Fig. 2. Expression of $D z D X R(A)$ and diosgenin content $(B)$ in different organs of $D$. zingiberensis. Data on $D z D X R$ expression in young tuber in April and in young leaf in October were absent due to that the tissues were not available. Means \pm SEs, $n=3$, different letters indicate significant differences at $P<0.01$.

\section{Discussion}

Some Dioscorea species produce significant quantities of diosgenin. For instances, diosgenin content in tubers of
D. composita and D. zingiberensis are usually over $3 \%$, even over 10 \% (Ding et al. 1981, Datta and Datta 1984, 
Hua et al 1989). The existence of large amounts of diosgenin in these plants implies that they can efficiently produce IPP and DAMPP for diosgenin biosynthesis.

A positive correlation between $D X R$ gene expression and production of isoprenoids has been observed in many species, such as Amomum villosum (Yang et al. 2012b), A. thaliana (Carretero-Paulet et al. 2002), Catharanthus roseus (Veau et al. 2000), Medicago truncatula (Bede et al. 2006), Populus $\times$ canescens (Mayrhofer et al. 2005), rose (Wang and Yao 2012), Salvia miltiorrhiza (Yang et al. 2012a), and transgenic peppermint (Mahmoud and Croteau 2001) and tobacco (Hasunuma et al. 2008, Zhang et al 2015). In present study, it was found that the expressions of $D z D X R$ did not match the amounts of diosgenin in the leaves and tubers of $D$. zingiberensis at all. But this does not mean that the MEP pathway is not involved in diosgenin biosynthesis in Dioscorea because secondary metabolites (intermediates and end products) can be transported from the synthesis sites to the other sites (Nagata et al. 2002, Burlat et al. 2004, Li and Hu 2009). Bennett et al. (1963) found that the shoot of D. spiculiflora was the major site of synthesis of phytosterols and sapogenins and believed that they were rapidly translocated to the tuber. Barker et al. (1966) also indicated that diosgenin was formed in the shoots of $D$. deltoidea and D. sylvatica and transported to the tubers. In fact, almost all of diosgenin in Dioscorea occurs in glycoside

\section{References}

Barker, E.A., Riartin, J.T., Wilson, A.P.: The distribution of diosgenin in Dioscorea spp. - Ann. appl. Biol. 58: 203-211, 1966.

Bede, J.C., Musser. R.O., Felton. G.W., Korth. K.L.: Caterpillar herbivory and salivary enzymes decrease transcript levels of Medicago truncatula genes encoding early enzymes in terpenoid biosynthesis. - Plant mol. Biol. 60: 519-531, 2006.

Bennett, R.D., Heftmann, E.: Biosynthesis of Dioscorea sapogenins from cholesterol. - Phytochemistry 4: 577-586, 1965.

Bennett, R.D., Heftmann, E., Joly, R.A.: Biosynthesis of diosgenin from 26-hydroxycholesterol in Dioscorea floribunda. - Phytochemistry 9: 349-353, 1970.

Bennett, R.D., Heftmann, E., Preston, W.H., Jr., Haun, J.R.: Biosynthesis of sterols and sapogenins in Dioscorea spiculiflora. - Arch. Biochem. Biophys. 103: 74-83, 1963.

Bick, J.A., Lange, B.M., Metabolic cross talk between cytosolic and plastidial pathways of isoprenoid biosynthesis: unidirectional transport of intermediates across the chloroplast envelope membrane. - Arch. Biochem. Biophys. 415: 146-154, 2003.

Burlat, V., Oudin, A., Courtois, M., Rideau, M., St-Pierre, B.: Co-expression of three MEP pathway genes and geraniol 10hydroxylase in internal phloem parenchyma of Catharanthus roseus implicates multicellular translocation of intermediates during the biosynthesis of monoterpene indole alkaloids and isoprenoid derived primary metabolites. - Plant J. 38:131141, 2004. form named dioscin, which undoubtedly can facilitate its movement. Recently, it was reported that the expression of the genes encoding squalene synthase (a crucial enzyme in biosynthesis of phytosterols) and 3-O-sterol glycosyltransferase (an enzyme catalyzing the first glycosylation step of diosgenin during dioscin biosynthesis) and the content of the first glycosylation product (diosgenin-3-O-glucoside) were much higher in the mature leaves than in the tubers of $D$. zingiberensis. These data strongly suggest that diosgenin was synthesized mainly in mature leaves of $D$. zingiberensis. Our results showed that during the growth period, $D z D X R$ was expressed consistently and significantly in mature leaves. This observation suggested that the MEP pathway was active in mature leaves of $D$. zingiberensis and significant amounts of IPP and DMAPP were formed there. Since transport of IPP and DMAPP produced by the MEP pathway from the plastids into the cytosol can occur in some plants, it was assumed that the MEP pathway might play an important role in the biosynthesis of diosgenin in D. zingiberensis. A further study on this assumption is necessary. One of strategies could be to produce transgenic $D$. zingiberensis plants over- and under-expressing $D z D X R$ and to analyze the relationship between $D z D X R$ expressions and diosgenin yields in transgenic and non-transgenic plants.
Cao, X., Zong, Z., Ju, X., Sun, Y., Dai, C., Liu, Q., Jiang, J.: Molecular cloning, characterization and function analysis of the gene encoding HMG-CoA reductase from Euphorbia pekinensis Rupr. - Mol. Biol. Rep. 37: 1559-1567, 2010.

Carretero-Paulet, L., Ahumada, I., Cunillera, N., RodríguezConcepción, M., Ferrer, A., Boronat, A., Campos, N.: Expression and molecular analysis of the Arabidopsis DXR gene encoding 1-deoxy-D-xylulose-5-phosphate reductoisomerase, the first committed enzyme of the 2-C-methyl-Derythritol 4-phosphate pathway. - Plant Physiol. 129: 15811591, 2002.

Chen, Y., Tang, Y.M., Yu, S.L., Han, Y.W., Kou, J.P., Liu, B.L., Yu, B.Y.: Advances in the pharmacological activities and mechanisms of diosgenin. - Chin. J. Nat. Med. 13: 578-587, 2015.

Cunningham, F.X., Pogson, B., Sun, Z., McDonald, K.A., DellaPenna, D., Gantt, E.: Functional analysis of $\beta$ and $\varepsilon$ lycopene cyclase enzymes of Arabidopsis reveals a mechanism for control of cyclic carotenoid formation. - Plant Cell 8: 1613-1626, 1996.

Datta, S.K., Datta, K.: Chemodifferentiation of diosgenin in Dioscorea composite. - Phytochemistry 33: 2684-2685, 1984.

Dewick, P.M. (ed): Medicinal Natural Products: A Biosynthetic Approach ( $3^{\text {rd }}$ Ed.). -John Wiley \& Sons, Chichester 2009.

Ding, Z., Zhou, L., Wang, Y., Tang, S.: Factors influencing diosgenin content of Dioscorea zingiberensis. - Chin. traditional herbal Drugs 12: 34-35, 1981. 
Dudareva, N., Andersson, S., Orlova, I., Gatto, N., Reichelt, M., Rhodes, D., Boland, W., Gershenson, J.: The nonmevalonate pathway supports both monoterpene and sesquiterpene formation in snapdragon flowers. - Proc. nat. Acad. Sci. USA 102: 933-938, 2005.

Gutensohn, M., Orlova, I., Nguyen, T.T., Davidovich-Rikanati, R., Ferruzzi, M.G., Sitrit, Y., Lewinsohn, E., Pichersky, E., Dudareva, N.: Cytosolic monoterpene biosynthesis is supported by plastid-generated geranyl diphosphate substrate in transgenic tomato fruits. - Plant J. 75: 351-363, 2013.

Hasunuma, T., Takeno, S., Hayashi, S., Sendai, M., Bamba, T., Yoshimura, S., Tomizawa, K., Fukusaki, E., Miyake, C.: Overexpression of 1-deoxy-D-xylulose-5-phosphate reductoisomerase gene in chloroplast contributes to increment of isoprenoid production. - J. Biosci. Bioeng. 105: 518-526, 2008

Heijne, V. G., Stepphuhn, J., Herrmann, R.G.: Domain structure of mitochondrial and chloroplast targeting peptides. - Eur. J. Biochem. 180: 535-545, 1989.

Hemmerlin, A., Harwood, J.L., Bach, T.J.: A raison d'être for two distinct pathways in the early steps of plant isoprenoid biosynthesis? - Proc. Lipid Res. 51: 95-148, 2012.

Hemmerlin, A., Hoeffler, J.F., Meyer, O., Tritsch, D., Kagan, I.A., Grosdemange-Billiard, C., Rohmer, M., Bach, T.J.: Cross-talk between the cytosolic mevalonate and the plastidial methylerythritol phosphate pathways in tobacco bright yellow-2 cells. - J. biol. Chem. 278: 26666-26676, 2003.

Hua, Z.P., Ding, Z.Z., He, S.A., Sheng, C.G.: Research on correlations between climatic factors and diosgenin content in Dioscorea zingiberensis. - Acta pharm. sin. 24: 702-706, 1989.

Huang, W., Zhao, H., Ni, J., Zuo, H., Qiu, L., Li., H., Li, H.: The best utilization of $D$. zingiberensis C.H. Wright by an ecofriendly process. - Bioresour. Tech. 99: 7407-7411, 2008.

Joly, R.A., Bonner, J., Benett, R.D., Heftmann, E.: Conversion of cholesterol to an open-chain saponin by Dioscorea floribunda. - Phytochemistry 8: 857-859, 1969a.

Joly, R.A., Bonner, J., Benett, R.D., Heftmann, E.: The biosynthesis of steroidal sapogenins in Dioscorea floribunda from doubly labelled cholesterol. - Phytochemistry 8: 1709$1711,1969 b$.

Laule, O., Furholz, A., Chang, H.S., Zhu, T., Wang, X., Heifetz, P.B., Gruissem, W., Lange, M.: Crosstalk between cytosolic and plastidial pathways of isoprenoid biosynthesis in Arabidopsis thaliana. - Proc. nat. Acad. Sci. USA 100: 68666871,2003

Li, J., Hu, Z.: Accumulation and dynamic trends of triterpenoid saponins in vegetative organs of Achyranthus bidentata. - J. integr. Plant. Biol. 51: 122-129, 2009.

Lichtenthaler, H.K.: Pathway of isoprenoid biosynthesis in plants. - Annu. Rev. Plant Physiol. Plant mol. Biol. 50: 47$65,1999$.

Mahmoud, S.S., Croteau, R.B.: Metabolic engineering of essential oil yield and composition in mint by altering expression of deoxyxylulose phosphate reductoisomerase and menthofuran synthase. - Proc. nat. Acad. Sci. USA. 98: 891-892, 2001.

Mayrhofer, S., Teuber, M., Zimmer, I., Louis, S., Fischbach, R.J., Schnitzler, J.P.: Diurnal and seasonal variation of isoprene biosynthesis-related genes in grey poplar leaves. - Plant Physiol. 139: 474-484, 2005.
Mou, Y., Zhou, K., Xu, D., Yu, R., Li, J., Yin, C., Zhou, L.: Enhancement of diosgenin production in plantlet and cell cultures of Dioscorea zingiberensis by palmarumycin $\mathrm{C}_{13}$ from the endophytic fungus, Berkleasmium sp. Dzf12. - Trop. J. Pharm. Res. 14: 241-248, 2015.

Nagata, N., Suzuki, M., Yoshida, S. and Muranaka, T.: Mevalonic acid partially restores chloroplast and etioplast development in Arabidopsis lacking the non-mevalonate pathway. - Planta 216: 345-350, 2002.

Patel, K., Gadewar, M., Tahilyani, V., Patel, DK.: A review on pharmacological and analytical aspects of diosgenin: a concise report. - Nat. Prod. Bioprospect. 2: 46-52, 2012.

Pfaffl, M.W.: A new mathematical model for relative quantification in real-time RT-PCR. - Nucl. Acids Res. 29: 2002-2007, 2001

Piironen, V., Lindsay, D.G, Miettinen, T.A., Toivo. J., Lampi, A.M.: Plant sterols: biosynthesis, biological function and their importance to human nutrition. - J. Sci. Food. Agr. 80: 939-966, 2000.

Schuhr, C.A., Radykewicz, T., Sagner, S., Latzel, C., Zenk, M.H., Arigoni, D., Bacher, A., Rohdich, F., Eisenreich, W.: Quantitative assessment of crosstalk between the two isoprenoid biosynthesis pathways in plants by NMR spectroscopy. - Phytochem. Rev. 2: 3-16, 2003.

Skorupinska-Tudek, K., Poznanski, J., Wojcik, J., Bienkowski, T., Szostkiewicz, I., Zelman-Femiak, M., Bajda, A. Chojnacki, T., Olszowska, O., Grunler, J. Meyer, O., Rohmer, M., Danikiewicz, W., Swiezewska, E.: Contribution of the mevalonate and methylerythritol phosphate pathways to the biosynthesis of dolichols in plants. - J. biol. Chem. 283: 21024-21035, 2008.

Stohs, S.J., Kaul, B., Staba, E.J.: The metabolism of ${ }^{14} \mathrm{C}-$ cholesterol by Dioscorea deltoidea suspension cultures. Phytochemistry 8: 1679-1686, 1969.

Suzuki, M., Muranaka, T.: Molecular genetics of plant sterol backbone synthesis. - Lipids 42: 47-54, 2007.

Veau, B., Courtois, M., Oudin, A., Chénieux. J.-C., Rideau, M., Clastre. M.: Cloning and expression of cDNAs encoding two enzymes of the MEP pathway in Catharanthus roseus. Biochim. biophys. Acta 1517: 159-163, 2000.

Vranová, E., Coman, D., Gruissem, W.: Network analysis of the MVA and MEP pathways for isoprenoid synthesis. - Annu. Rev. Plant Biol. 64: 665-700, 2013.

Wang, H., Yao, L.: Cloning and expression profile of 1-deoxyD-xylulose 5-phosphate reductoisomerase gene from an oilbearing rose. - Russ. J. Plant Physiol. 61: 548-455, 2014.

Yang, D., Du, X., Liang, X., Han, R., Liang, Z., Liu, Y., Liu, F., Zhao, J.: Different roles of the mevalonate and methylerythritol phosphate pathways in cell growth and tanshinone production of Salvia miltiorrhiza hairy roots. PLoS ONE 7: e46797, 2012a.

Yang, J., Adhikari, M.N., Liu, H., Xu, H., He, G., Zhan, R., Wei, J., Chen, W.: Characterization and functional analysis of the genes encoding 1-deoxy-D-xylulose-5-phosphate reductoisomerase and 1-deoxy-D-xylulose-5-phosphate synthase, the two enzymes in the MEP pathway, from Amomum villosum Lour. - Mol. Biol. Rep. 39: 8287-8296, 2012 b.

Zhang, H., Niu, D., Wang, J., Zhang, S., Yang, Y, Jia H, Cui, H.: Engineering a platform for photosynthetic pigment, hormone and cembrane-related diterpenoid production in Nicotiana tabacum. Plant Cell Physiol. 56: 2125-2138, 2015. 